\title{
Refractometric sensors based on multimode interference in a thin-film coated single-mode- multimode-single-mode structure with reflection configuration
}

\author{
Ignacio Del Villar*, Abian B. Socorro, Jesus M. Corres, Francisco J. Arregui, and Ignacio R. Matias \\ Electrical and Electronic Engineering Department, Public University of Navarra, 31006 Pamplona, Spain \\ *Corresponding author:ignacio.delvillar@unavarra.es
}

Thin-film coated single-mode-multimode-single-mode (SMS) structures have been analyzed both theoretically and experimentally with the aim of detecting different refractive indices. By adequate selection of the thickness of the thin-film and of the diameter of the multimode segment in the SMS structure a seven-fold improvement can be obtained in the sensitivity of the device to the surrounding medium refractive index, achieving a maximum sensitivity of $1199.18 \mathrm{~nm} /$ refractive index unit for the range of refractive indices from 1.321 to 1.382 . The fact of using layer-by-layer self-assembly for deposition both on the cladding and on the tip of the multimode segment permits to increase the reflected power, which avoids the application of a mirror on the tip of the multimode segment. (c) 2013 Optical Society of America

OCIS codes: 060.2370, 240.0310, 120.3180

\section{INTRODUCTION}

The research field of optical fiber sensors has experienced a significant advance during the last decade thanks to the application of nanodeposition techniques towards the development of thin-film coated devices [1-3]. The detection of parameters based on the modification of the intrinsic properties of the optical fiber (i.e. strain or temperature) [4], has been expanded to any parameter that induces a change in the thinfilm and, consequently, a variation in the power transmitted through the optical fiber [5-7]. Moreover, the discovery that the adequate selection of the parameters of the thin-film permits to increase the sensitivity of the device has become a hot topic in subdomains such as cladding removed multimode optical fiber [8], or long-period fiber gratings (LFPGs) [9-10]. Recently, a well known structure, multimode interference based single-modemultimode-single-mode structure (SMS) [11-17], has been explored in terms of influence of thin-film deposition [18-19], with a wavelength shift of the resonance bands that resembles that observed in long-period fiber gratings. The rules for optimization are given in [20] and an optimized $\mathrm{pH}$ sensor can be found in [21].

However, publications based on thin-film coated SMS structures use a transmission configuration (a multimode segment spliced to two single mode fibers) [18-21], which reduces its easiness to handle and, hence, the commercial utilization of this device. In this work a simpler reflection configuration is used instead (the multimode segment is only spliced to one single mode segment). Though there are some previous works of multimode interference in SMS structures in reflection configuration [22-24], this is the first time to our knowledge that thin-film SMS coated devices in a reflection configuration are explored. Moreover, the sensitivity to the surrounding medium refractive index (SRI) is analyzed both theoretically and experimentally for different diameters of the multimode section and different thin-film thickness, confirming the theoretical predictions of [20].

\section{THEORY}

Propagation of light in an SMS structure with transmission configuration (see Fig. 1a) is based on coupling of light from the input single-mode fiber (SMF) to several modes in the multimode fiber (MMF) segment [12-17]. Light propagates through different modes in the MMF segment and, hence, the phase of the modes is different at the point where light is coupled to the output SMF segment.

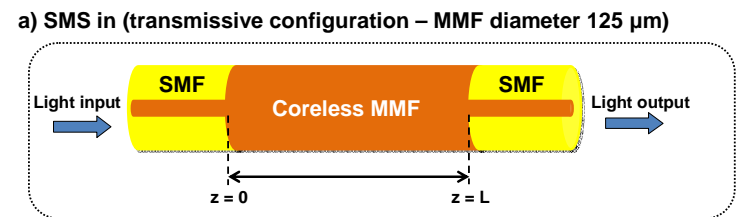

b) SMS in (reflective configuration - MMF diameter $125 \mu \mathrm{m}$ )

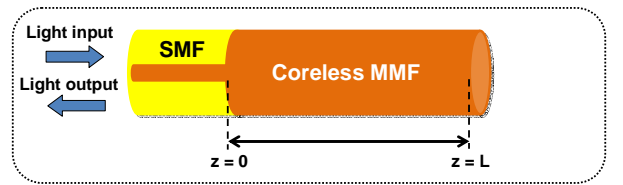

c) SMS in (reflective configuration - MMF diameter $61.5 \mu \mathrm{m}$ )

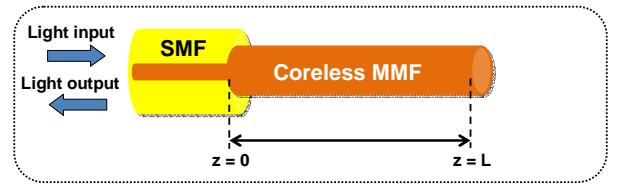

Fig. 1: Set-ups for multimode interference based single-mode-multimodesingle-mode structure (SMS). 
The coupling coefficient reaches a peak when the length of the MMF segment is selected so that the field distribution at the end of the MMF is an image of the input field [11]. This is called by other authors as the self-imaging effect (a light field condensation between the several guided modes in a particular plane), distinguishing it from other maxima obtained by a fractional constructive interference [17].

The self-imaging effect permits to obtain the highest transmission in the optical spectrum and its central wavelength can be tuned by adequate selection of the parameters of the following expression [17]:

$$
Z_{i}=\frac{4 D^{2} n}{\lambda}
$$

where $D$ and $n$ are the MMF diameter and refractive index, respectively, and $\lambda$ is the operational wavelength.

The expression for an SMS structure in reflection configuration (see Fig. $1 \mathrm{~b}$ and 1c) can be obtained just by dividing expression (1) by 2 :

$$
Z_{i}=\frac{2 D^{2} n}{\lambda}
$$

However, in order to obtain the same response with the reflection configuration a mirror is required [24], which can be avoided at a cost of a power loss [22-23].

As an example in Fig. 2 two SMS structures are compared. For the sake of comparison, the aim will be to set the self-image effect band at the same wavelength. In [17,19-21] the self-image band was located at $1550 \mathrm{~nm}$, which is the third optical communications window. However, in view of the spectral range that can be monitored with the experimental set-up explained in section 3 , and in view of the wavelength shift to the red that occurs after deposition of a thin-film or by increasing the surrounding medium refractive index, it has been decided to locate the self-image band at $1500 \mathrm{~nm}$. According to the numerical analysis obtained with FIMMWAVE ${ }^{\mathbb{R}}$, the SMS structure in transmission configuration requires a $60.4 \mathrm{~mm}$ long MMF section length, whereas the SMS structure in reflection configuration requires a $30.2 \mathrm{~mm}$ long MMF section. For the simulations the refractive index of both the MMF section and the cladding of the SMF section is that of silica [25], whereas the refractive index of the core of the SMF section is that of silica multiplied by 1.0036 (according to the specifications of the provider of the SMF, Telnet Redes Inteligentes S.A, Spain).

The simulation results (see Fig. 2) agree well with the expressions 1 and 2, which indicate the length of both transmission and reflection configuration must be $60.25 \mathrm{~mm}$ and $30.125 \mathrm{~mm}$ respectively in order to set self-image effect band at $1500 \mathrm{~nm}$.

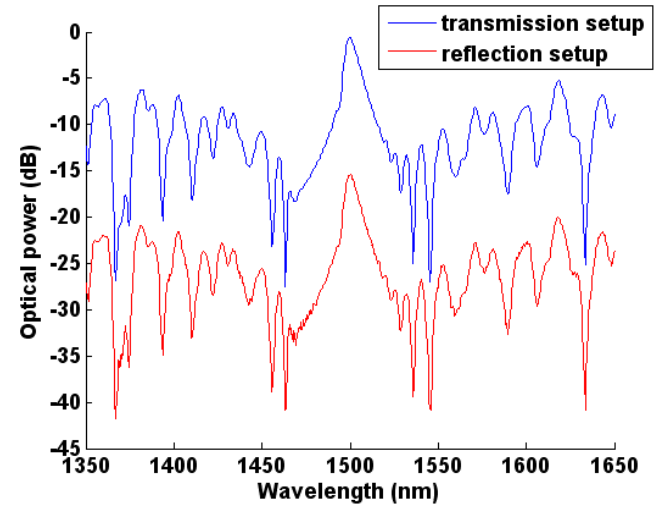

Fig. 2: Numerical results: optical power transmitted and reflected respectively with a transmission configuration set-up (60 mm long MMF section) and with a reflection configuration set-up (30 mm long MMF section).

\section{EXPERIMENTAL SECTION}

\section{Device fabrication}

Three SMS structures in reflection configuration were fabricated by splicing a segment of coreless multimode fiber (MMF) from POFC Inc. to a standard SMF pigtail from Telnet Redes Inteligentes Inc. In two of the structures, the diameter of the MMF section was $125 \mu \mathrm{m}$ and the length was $30 \mathrm{~mm}$ (see the set-up of Fig. 1b), whereas in the other one the diameter was 61.5 $\mu \mathrm{m}$ and the length $15 \mathrm{~mm}$ (see the set-up of Fig. 1c). These values were selected to satisfy that the central wavelength of the selfimage band is located approximately at $1500 \mathrm{~nm}$. In order to corroborate the numerical results of Fig 2, also an SMS structure in transmission configuration (the set-up of Fig. 1a) with a 60 $\mathrm{mm}$ long MMF section was fabricated. The results of both the SMS structure in transmission configuration and one of the SMS structures in reflection configuration (MMF section length 30 $\mathrm{mm}$ and diameter $125 \mu \mathrm{m}$ ), are represented in Fig. 3. The wavelength shift to the blue of the experimental results if compared to the numerical ones in Fig. 2 is due to inaccuracies in obtaining a MMF segment of exactly $60.4 \mathrm{~mm}$ for the transmission configuration device and $30.2 \mathrm{~mm}$ for the reflection configuration device, which would lead to a self-image band exactly centered at $1500 \mathrm{~nm}$.

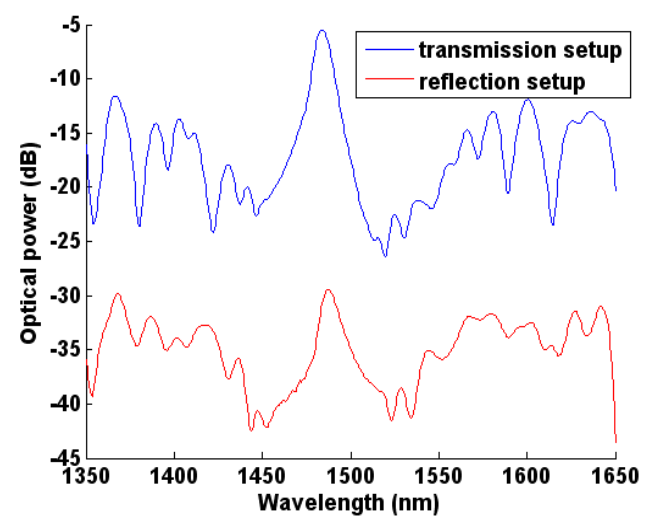

Fig. 3: Experimental results: optical power transmitted and reflected respectively with a transmission configuration set-up (60 mm long MMF section) and with a reflection configuration set-up (30 mm long MMF section).

For the deposition of the thin-film, layer-by-layer assembly process (LbL) was used, which consists of the deposition of bilayers by electrostatic attraction [26]. Each bilayer is composed of a positively and a negatively charged monolayer. For positively charged monolayer a solution of Titanium (IV) oxide nanoparticles in ultrapure water was used, whereas for the negatively charge monolayer a poly(sodium 4-styrenesulfonate) PSS water solution was used [27]. The $\mathrm{pH}$ of both anionic and cationic solutions was adjusted to 2.0 .

The coating fabrication begins with the dipping of the optical fiber device into the $\mathrm{TiO}_{2}$ nanoparticles solution during 2 minutes. After this step, the substrate is rinsed 1 minute in acid 
water and air-dried during 30 seconds. These steps are repeated with the PSS solution. This process is repeated up to 2 bilayers and 5 bilayers respectively for the SMS structures with MMF section of diameter $125 \mu \mathrm{m}$, and up to 5 bilayers for the SMS structures with MMF section of diameter $61.5 \mu \mathrm{m}$

All the chemicals used were purchased from Sigma-Aldrich Inc. and used without further purification. All the measurements were performed at room temperature $\left(25^{\circ} \mathrm{C}\right)$.

\section{Device characterization}

The reflectance spectra are collected after each bilayer using the experimental set-up shown in Fig. 4. It consists of a broadband light source (Agilent 83437A) connected to one end of a single-mode fiber $2 \times 2$ coupler. The second end is connected to an Agilent 86140B optical spectrum analyzer. The third end is connected to the sensor pigtail, and the last end is surrounding with an index matching gel. This allows covering a wide range of wavelengths, from 1150 to $1680 \mathrm{~nm}$. In order to eliminate any variations or artifacts coming from the source, the obtained spectra were normalized to the source spectrum.

After the fabrication of the sensor, the same set-up was used to characterize the device when it is subjected to changes in the external RI. In order to observe the wavelength shift of the selfimaging band, the sensitive fragment is immersed in different glycerol in water solutions: $0 \%, 10 \%, 20 \%, 30 \%, 40 \%, 50 \%$ and $60 \%$; which respectively correspond to refractive index values: $1.321,1.336,1.352,1.367,1.382,1.397$ and 1.413 [28,29].

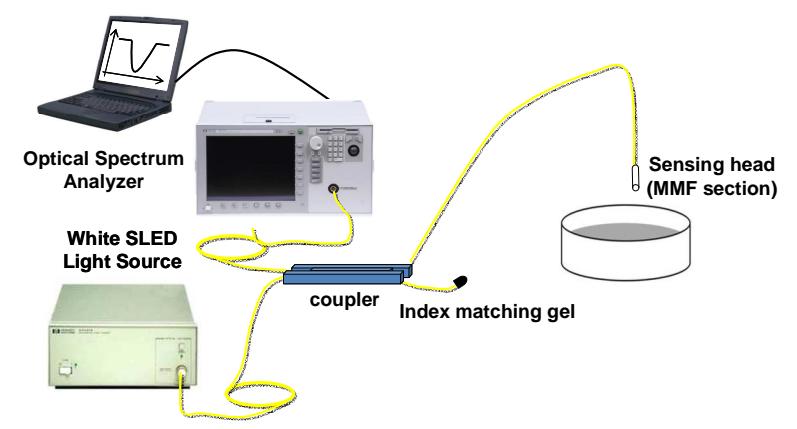

Fig. 4: Experimental set-up in reflection configuration for monitoring of the spectral variations during the deposition process and at introducing the sensor in different refractive index solutions.

\section{RESULTS}

As it was indicated in the previous section the three SMS structures are coated with a $\mathrm{TiO}_{2} / \mathrm{PSS}$ thin film. The refractive index dispersion model can be found in [27], where the values from 1150 to $1680 \mathrm{~nm}$ range from $1.749+0.011$ to $1.737+0.005 \mathrm{i}$ according to [27]. The bilayer thickness is $17 \mathrm{~nm}$. This value was obtained with the estimation method described in [30]. With these parameters, in Fig. 5 the evolution of the spectrum as a function of the number of bilayers can be observed for the three devices in reflection configuration. In Fig. 6, the experimental results corresponding to the theoretical predictions of Fig. 5 are presented. The device with MMF section of diameter $61.5 \mu \mathrm{m}$ experiments a higher wavelength shift. At the same time the reflected power increases because also the tip of the MMF section is coated. The deposition at the tip induces an interferometric cavity $[31,32]$. This causes a sinus plot as a function of the cavity thickness whose amplitude decreases due to the absorbance. In view that the refractive index of the cavity is higher than both the optical fiber and the surrounding medium, there is an increase of the reflected optical power as a function of the number of bilayers deposited (the sinus plot evolves from 0 to $\Pi / 2$ ) [31]. The number of bilayers deposited in this work is low enough to guarantee that the sinus plot does not start to decrease (phase $\Pi / 2$ to $3 \Pi / 2$ ). Consequently, the bilayers deposited on the MMF section tip induce an increase in the reflected power at the same time the resonances are blue shifted.

In addition to this, the width of the resonance increases slightly. This is explained by an increase in the evanescent field of modes when a high refractive index thin-film is deposited on a multimode structure [9], which actually occurs for modes in the MMF section. This phenomenon is similar to that observed when the diameter of the MMF section in an SMS structure is reduced. $[16,17]$. The self-image band broadens, which coincides with a higher interaction of modes with the surrounding medium [17].

After the fabrication process the SMS structures were tested against the surrounding medium refractive index by immersing them in different glycerol in water solutions, as indicated in section 3. In Fig. 7 and 8 theoretical and experimental results are presented.

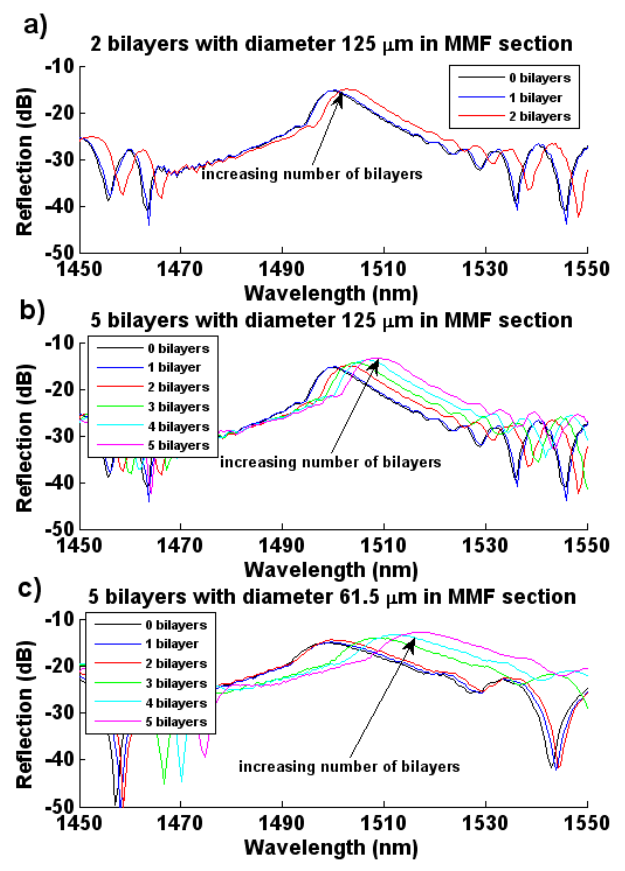

Fig. 5: Spectral evolution (numerical results) with progressive deposition of: a) 2 bilayers in an SMS with MMF section length $30 \mathrm{~mm}$ and diameter $125 \mu \mathrm{m}$, b) 5 bilayers in an SMS with MMF section length $30 \mathrm{~mm}$ and diameter $125 \mu \mathrm{m}$, c) 5 bilayers in an SMS with MMF section length 15 $\mathrm{mm}$ and diameter $61.5 \mathrm{\mu m}$. 
a)

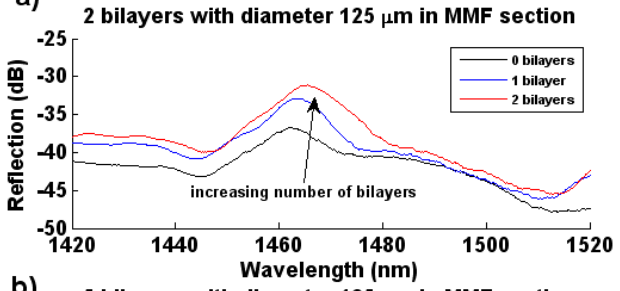

b) 5 bilayers with diameter $125 \mu \mathrm{m}$ in MMF section

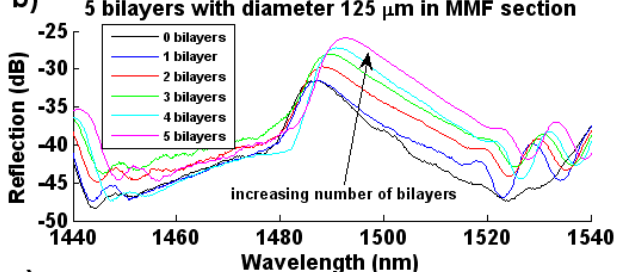

c) 5 bilayers with diameter $61.5 \mu \mathrm{m}$ in MMF section

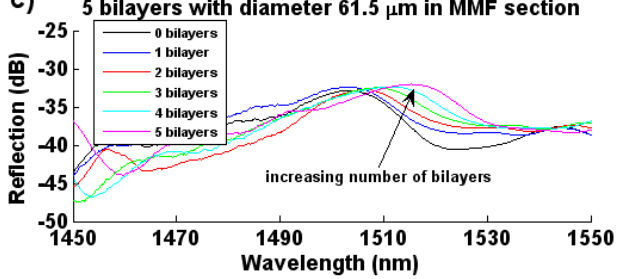

a)

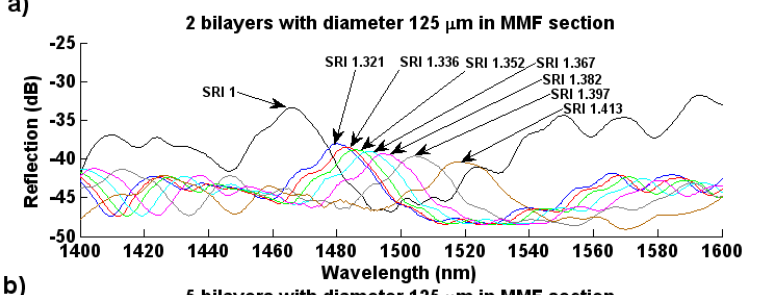

b)

5 bilayers with diameter $125 \mu \mathrm{m}$ in MMF section

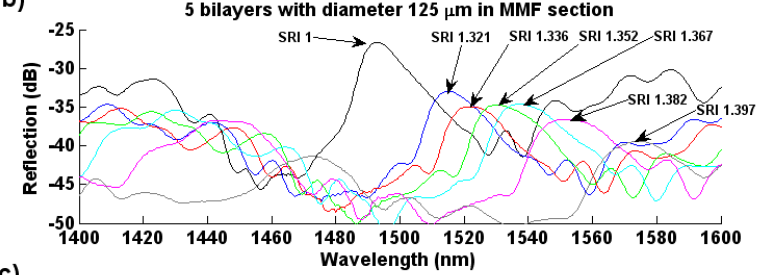

c)

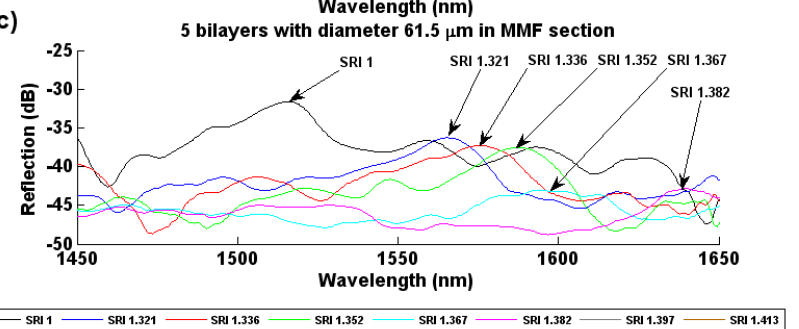

Fig. 6 Spectral evolution (experimental results) with progressive deposition of: a) 2 bilayers in an SMS with MMF section length $30 \mathrm{~mm}$ and diameter $125 \mu \mathrm{m}$, b) 5 bilayers in an SMS with MMF section length $30 \mathrm{~mm}$ and diameter $125 \mu \mathrm{m}$, c) 5 bilayers in an SMS with MMF section length $15 \mathrm{~mm}$ and diameter $61.5 \mathrm{\mu m}$.

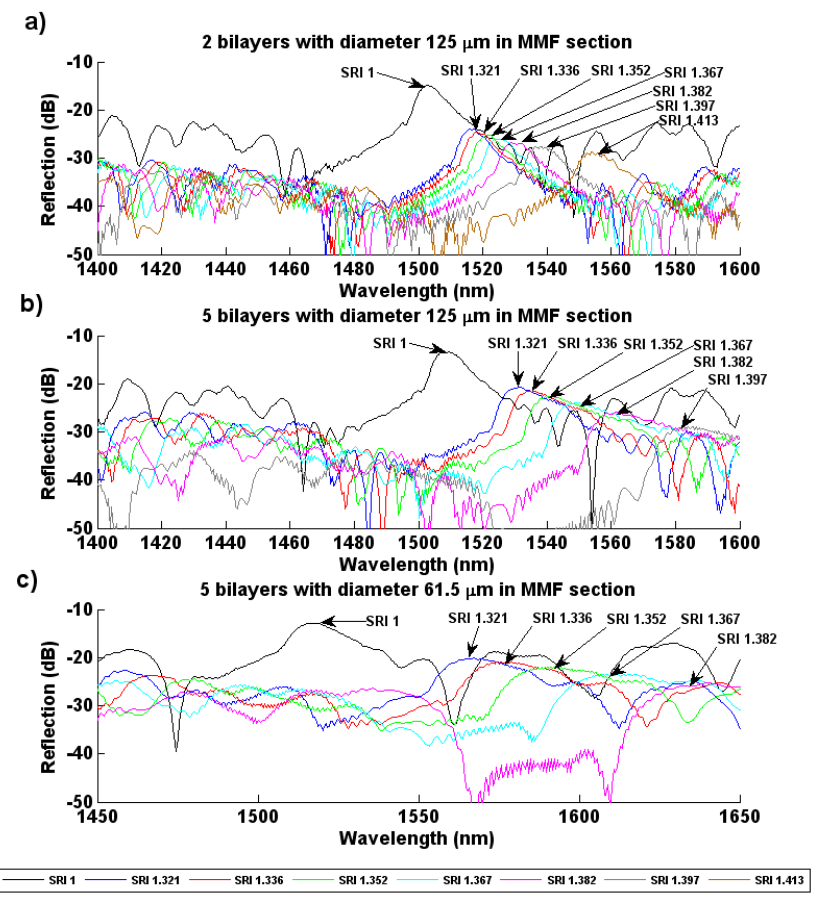

Fig. 7: Numerical results of response to different surrounding refractive indices (SRI). The self-imaging band is highlighted with an arrow. a) SMS with MMF section length $30 \mathrm{~mm}$ and diameter $125 \mu \mathrm{m}$ coated with 2 bilayers in an b) SMS with MMF section length $30 \mathrm{~mm}$ and diameter 125 um coated 5 bilayers c) SMS with MMF section length $15 \mathrm{~mm}$ and diameter $61.5 \mu \mathrm{m}$ coated with 5 bilayers.
Fig. 8: Experimental results of response to different surrounding refractive indices (SRI). The self-imaging band is highlighted with an arrow. a) SMS with MMF section length $30 \mathrm{~mm}$ and diameter $125 \mu \mathrm{m}$ coated with 2 bilayers in an b) SMS with MMF section length $30 \mathrm{~mm}$ and diameter 125 $\mu \mathrm{m}$ coated 5 bilayers c) SMS with MMF section length $15 \mathrm{~mm}$ and diameter 61.5 um coated with 5 bilayers.

In Fig. 9 the evolution of the central wavelength of the selfimaging band is represented both numerically and experimentally as a function of the refractive index for the structures analyzed in Fig. 7 and 8 and for the same structures without deposition. The results reveal the improvement of the sensitivity if more bilayers are deposited ( 5 bilayers vs 2 bilayers) and if the diameter of the MMF section is reduced $(125 \mu \mathrm{m}$ vs $61.5 \mu \mathrm{m})$. This first question is explained in [20], whereas the second question is detailed in [17], where it is indicated that SMS structures with lower MMF section diameter are more sensitive to RI variations because the interaction between the interfered high-order modes and the external medium is increased.

In order to have a more precise idea of the improvement, in Table 1 the sensitivity (wavelength shift vs a refractive index range from 1.321 to 1.382) is indicated for both structures with and without thin-film. In the experimental results, a 3.9-factor increase is obtained with the 5 bilayers thin-film deposited SMS structure with MMF segment diameter $125 \mu \mathrm{m}$, if compared with the same structure without thin-film. This value is exactly the same obtained when the 5 bilayers thin-film coated SMS structure with MMF segment diameter $61.5 \mu \mathrm{m}$ is compared with the same structure without thin-film. However, if the sensitivities of structures with MMF segment diameter $61.5 \mu \mathrm{m}$ are compared to those with MMF segment diameter $125 \mu \mathrm{m}$ an improvement factor of 1.9 is obtained. In other words, if these two factors are multiplied, the structure with the best performance (the 5 bilayers thin-film coated SMS structure with MMF segment diameter $61.5 \mu \mathrm{m}$ ) with the structure with the worst performance (the uncoated SMS structure with MMF segment diameter $125 \mu \mathrm{m}$ ) a improvement factor of 7.4 is obtained. 

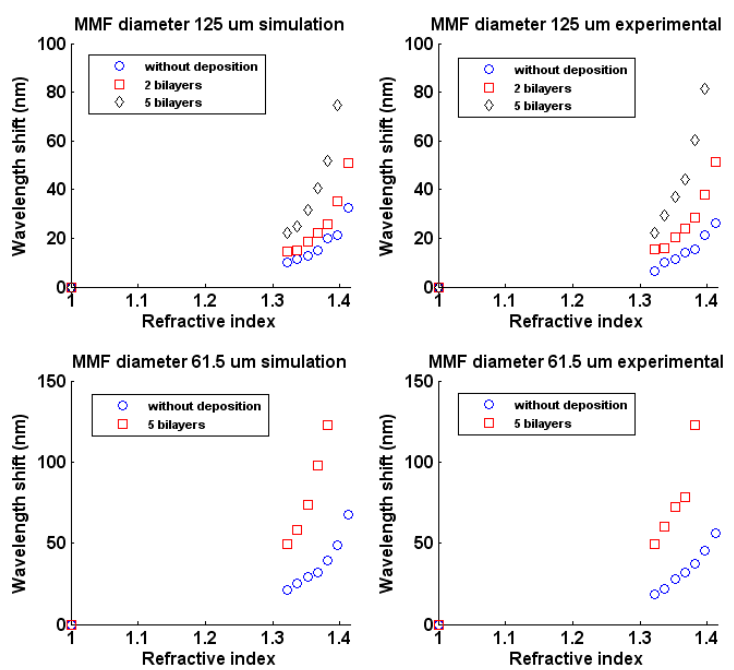

Fig. 9: Theoretical and experimental wavelength shift of the central wavelength of the self-imaging band for different surrounding refractive indices (SRI).

\begin{tabular}{|c|c|c|}
\hline & $\begin{array}{c}\text { Theory } \\
\text { (nm/RIU) }\end{array}$ & $\begin{array}{c}\text { Experiment } \\
\text { (nm/RIU) }\end{array}$ \\
\hline Diameter $125 \mu \mathrm{m}$ and no thin-film & 163.93 & 162.30 \\
\hline Diameter $125 \mu \mathrm{m}$ and thin-film (2 bilayers) & 188.52 & 216.39 \\
\hline Diameter $125 \mu \mathrm{m}$ and thin-film (5 bilayers) & 491.80 & 631.15 \\
\hline Diameter $61.5 \mu \mathrm{m}$ and no thin-film & 295.08 & 306.56 \\
\hline Diameter $61.5 \mu \mathrm{m}$ and thin-film (5 bilayers) & 1196.72 & 1199.18 \\
\hline
\end{tabular}

Table 1: Sensitivity (wavelength shift in nanometers vs refractive index variation from 1.321 to $1.382 \mathrm{in} \mathrm{nm} / \mathrm{RIU}$ ) for the different SMS structures analyzed.

The selection of the refractive index range used for analyzing the sensitivity (1.321 to 1.382 ) requires an explanation. This range is due to the detection limit of the explored devices and it depends on the number of bilayers deposited. As the number of bilayers (the thickness of the thin-film) increases, the self-image band is shifted but at the same time it approaches a fading region where the self-image band is no longer visible [20]. This is caused by the lossy-mode resonance phenomenon [8]. The two devices with 5 bilayers are closer to the fading region, and also due to the higher broadening of SMS structures with an MMF section of lower diameter, the limit should be set by the SMS with MMF section length $15 \mathrm{~mm}$ and diameter $61.5 \mathrm{\mu m}$ coated with 5 bilayers. This assumption agrees with the results shown in Fig. 7, where the limit of detection is 1.382 for this structure (higher refractive indices lead to spectra where the self-image band was not visible and are not shown). The value 1.382 is lower than the limit of the other two structures (for the SMS with MMF section length $30 \mathrm{~mm}$ and diameter $125 \mu \mathrm{m}$ coated 5 bilayers it is 1.397 and for the SMS with MMF section length 30 $\mathrm{mm}$ and diameter $125 \mu \mathrm{m}$ coated 2 bilayers it is at least 1.413 , the highest refractive index explored in this work).

Finally, in order to prove the long-term repeatability of the devices developed in this work, the response after 2 months of the SMS device with MMF section of length $30 \mathrm{~mm}$, diameter 125 $\mu \mathrm{m}$ and coated with 5 bilayers is presented in Fig. 10. Initially the sensor was surrounded by air (SRI=1) and after that it was immersed several times in water (SRI $=1.321)$ and $30 \%$ glycerol in water solution (SRI=1.367). The central wavelength of the selfimage band is 1493, 1515 and $1533 \mathrm{~nm}$ for the three refractive indices analyzed, which agrees with the values obtained in Fig. 8b: 1493,1515 and 1537 . The error in the last value may be caused by the utilization of a new glycerol solution different to that used for the experiments of Fig. 8b.

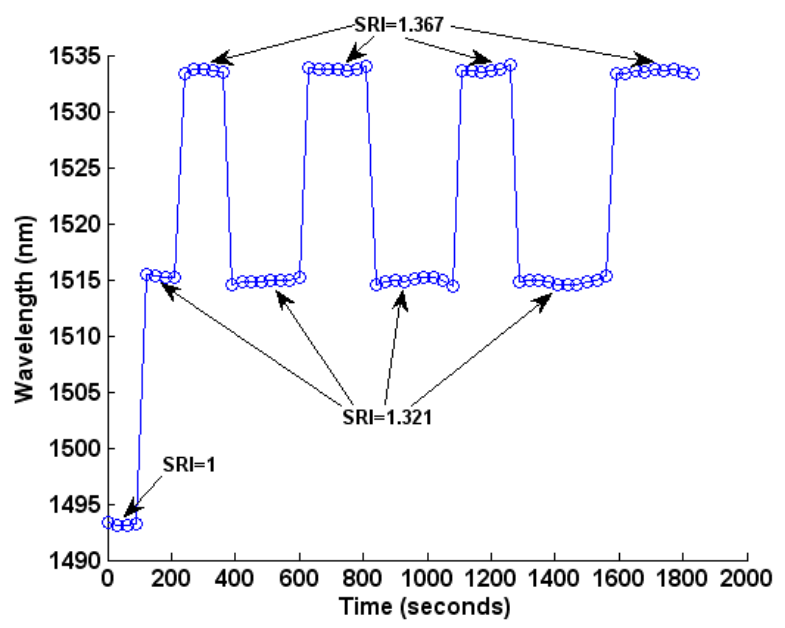

Fig. 10: Response of a SMS device with a MMF section of length $30 \mathrm{~mm}$, diameter $125 \mu \mathrm{m}$ and coated with 5 bilayers two months after the fabrication of the sensor

\section{CONCLUSIONS}

In this work, it has been proved both theoretically and experimentally that the application of a thin-film on an SMS structure permits to increase the sensitivity of the device by almost a factor of 4 . This factor can even be increased if a multimode segment with a small diameter is chosen. In this work by comparing multimode segments of 125 and $61.5 \mu \mathrm{m}$ it has been obtained nearly a two-fold improvement, which combined with the application of a thin-film, permits to obtain more than a seven-fold sensitivity improvement.

The results agree with those reported in [20], where a transmission configuration was used. However, in this work a reflective configuration has been used, which permits to improve the portability and easiness to handle of the devices developed. Moreover, the application of the thin-film not only to the cladding of the multimode segment but also on the tip has permitted to obtain a higher reflected power due to interferometry at the formed nanocavity, which permits to avoid the need of a mirror on the tip.

The results obtained for detection of surrounding medium refractive indices and the possibility of deposition of material sensitive to chemical species indicate the adequateness of SMS structures in reflective configuration for applications in domains such as chemistry or biology.

\section{ACKNOWLEDGEMENTS}

This work was supported in part by the Spanish Ministry of Education and Science-FEDER TEC2010-17805 and Government of Navarra research grants. 


\section{REFERENCES}

1. C. R. Zamarreño, J. M. Corres, J. Goicoechea, I. Del Villar, I. R. Matias and F. J. Arregui, "Fiber Optic Nanosensors," in Optochemical Nanosensors, A. Cusano, F. J. Arregui, M. Giordano, A. Cutolo, Taylor and Francis (2012), pp. 167203.

2. T. Grujic, B. T. Kuhlmey, A. Argyros, S. Coen and C. M. De Sterke, "Solid-core fiber with ultra-wide bandwidth transmission window due to inhibited coupling," Opt. Express 18, 25556-25566 (2010).

3. C. Markos, S. N. Yannopoulos and K. Vlachos, "Chalcogenide glass layers in silica photonic crystal fibers," Opt. Express 20, 14814-14824 (2012).

4. B. Lee, "Review of the present status of optical fiber sensors," Opt. Fiber Technol. 9, 57-79 (2003).

5. C. R. Zamarreño, M. Hernáez, I. Del Villar, I. R. Matias and F. J. Arregui, "Tunable humidity sensor based on ITOcoated optical fiber," Sens. Actuators B 146, 414-417, (2010).

6. C. Markos, W. Yuan, K. Vlachos, G. E. Town and Ole Bang. "Label-free biosensing with high sensitivity in dual-core microstructured polymer optical fibers," Opt. Express 19, 7790-7798, (2011).

7. T. Wang, S. Korposh, S. James, R. Tatam and S.-W. Lee, "Optical fibre long period grating sensor with a polyelectrolyte alternate thin film for gas sensing of amine odors," Sens. Actuators B, 185, 117-124, (2013).

8. I. Del Villar, M. Hernáez, C. R. Zamarreño, P. Sanchez, C. Fernandez-Valdivielso, F. J. Arregui and I. R. Matías, "Design Rules for Lossy Mode Resonance Based Sensors," Appl. Opt. 51, 4298-4307 (2012).

9. I. Del Villar, I. R. Matias, F. J. Arregui and P. Lalanne, "Optimization of sensitivity in long period gratings with overlay deposition," Opt. Express 13, 56-69 (2005).

10. P. Pilla, C. Trono, F. Baldini, F. Chiavaioli, M. Giordano and A. Cusano, "Giant sensitivity of long period gratings in transition mode near the dispersion turning point: an integrated design approach," Opt. Lett. 37, 4152-4154 (2012).

11. A. Mehta, W. Mohammed and E. G. Johnson, "Multimode interference-based fiber-optic displacement sensor," IEEE Photon. Technol. Lett. 15, 1129-1131 (2003).

12. W. S. Mohammed and P. W. E. Smith, "All-fiber multimode interference bandpass filter," Opt. Lett. 31, 2547-2549, (2006).

13. J. E. Antonio-Lopez, A. Castillo-Guzman, D. A. MayArrioja, R. Selvas-Aguilar and P. LiKamWa, "Tunable multimode-interference bandpass fiber filter," Opt. Lett. 35, 324-326 (2010).

14. Q. Wu, Y. Semenova, A. M. Hatta, P. Wang and G. Farrell, "Single-mode-multimode-singlemode fiber structures for simultaneous measurement of strain and temperature," Microw. And Opt. Tech. Lett. 53, 21812185, (2011)

15. V. I. Ruiz-Pérez, M. A. Basurto-Pensado, P. LiKamWa, J. J. Sánchez-Mondragón, D. A. May-Arrioja, "Fiber Optic Pressure Sensor using Multimode Interference," J. Phys.: Conf. Ser. 274, 012025 (2011).

16. Q. Wu, Y. Semenova, P. Wang and G. Farrell, "High sensitivity SMS fiber structure based refractometer analysis and experiment," Opt. Express 19, 7937-7944 (2011).

17. S. Silva, E. G. P. Pachon, M. A. R. Franco, J. G. Hayashi, F. X. Malcata, O. Frazão, P. Jorge and C. M. B. Cordeiro, "Ultrahigh-sensitivity temperature fiber sensor based on multimode interference," Appl. Opt. 51, 3236-3242, (2012).
18. L. L. Xue and L. Yang, "Sensitivity enhancement of RI sensor based on SMS fiber structure with high refractive index overlay," J. Lightwave. Technol. 30, 1463-1469 (2012).

19. A. B. Socorro, I. Del Villar, J. M. Corres, F. J. Arregui and I. R. Matias, "Mode transition in complex refractive index coated single-mode-multimode-single-mode structure," Opt. Express 21, 12668-12682 (2013).

20. I. Del Villar, A. B. Socorro, J. M. Corres, F. J. Arregui and I. R. Matias, "Optimization of Sensors Based on Multimode Interference in Single-Mode-Multimode-Single-Mode Structure", J. Lightwave Technol. 31, 3460-3468 (2013).

21. A.B. Socorro, I. Del Villar, J. M. Corres, F. J. Arregui and I. R. Matias, "Sensitivity enhancement in a multimode interference-based SMS fibre structure coated with a thinfilm: Theoretical and experimental study," Sens. Actuators B 190, 363- 369 (2014).

22. E. Li, X. Wang and C. Zhang, "Fiber-optic temperature sensor based on interference of selective higher-order modes," Appl. Phys. Lett. 89, 09119 (2006).

23. O. Frazão, S. O. Silva, J. Viegas, L. A. Ferreira, F. M. Araújo and J. L. Santos, "Optical fiber refractometry based on multimode interference," Appl. Opt. 50, 184-188, (2011).

24. J. E. Antonio-Lopez, J. J. Sanchez-Mondragon, P. LiKamWa, and D. A. May-Arrioja "Fiber-optic sensor for liquid level measurement," Opt. Lett. 36, 3425-3427, (2011).

25. G. P. Agrawal, "Nonlinear fiber optics" (3rd ed., Academic Press: New York, 2001), p. 8.

26. G. Decher, "Fuzzy Nanoassemblies: Toward Layered Polymeric Multicomposites," Science 277, 1232-1237 (1997).

27. M. Hernáez, I. Del Villar, C. R. Zamarreño, F. J. Arregui and I. R. Matías "Optical fiber refractometers based on lossy mode resonances supported by TiO2 coatings," Appl. Opt. 49, 3980-3985, (2010).

28. P. R. Cooper, "'Refractive-index measurements of liquids used in conjunction with optical fibers," Appl. Opt. 22, 3070-3072, (1983).

29. D. Masahiko and A. Masumura "Measurement of the refractive index of distilled water from the near-infrared region to the ultraviolet region," Appl. Opt. 46, 3811-3820 (2007)

30. I. Del Villar, I. R. Matias, F. J. Arregui, and R. O. Claus, "Fiber-optic hydrogen peroxide nanosensor," IEEE Sens. J. 5, 365-371 (2005).

31. F. J. Arregui, I. R. Matías, Y. Liu, K. M. Lenahan and R. O. Claus "Optical fiber nanometer-scale Fabry-Perot interferometer formed by the ionic self-assembly monolayer process," Opt. Lett. 24, 596-598 (1999).

32. C. E. Lee, W. N. Gibler, R. A. Atkins, and H. F. Taylor, "In-line fiber Fabry-Perot interferometer with highreflectance internal mirrors" J. Lightwave Technol. 10, 1376-1379 (1992). 